OPEN ACCESS

Edited by:

Hana Burianova,

Swansea University, United Kingdom

Reviewed by:

Mathieu Wolff,

Centre National de la Recherche Scientifique (CNRS), France Deniz Yilmazer-Hanke, University of UIm, Germany

${ }^{*}$ Correspondence: Anna S. Mitchell anna.mitchell@psy.ox.ac.uk

Received: 01 January 2019 Accepted: 17 June 2019 Published: 03 July 2019

Citation:

Perry BAL and Mitchell AS (2019) Considering the Evidence for Anterior and Laterodorsal Thalamic Nuclei as Higher Order Relays to Cortex.

Front. Mol. Neurosci. 12:167. doi: 10.3389/fnmol.2019.00167

\section{Considering the Evidence for Anterior and Laterodorsal Thalamic Nuclei as Higher Order Relays to Cortex}

\author{
Brook A. L. Perry and Anna S. Mitchell * \\ Department of Experimental Psychology, University of Oxford, Oxford, United Kingdom
}

Our memories are essential in our daily lives. The frontal and cingulate cortices, hippocampal system and medial temporal lobes are key brain regions. In addition, severe amnesia also occurs after damage or dysfunction to the anterior thalamic nuclei; this subcortical thalamic hub is interconnected to these key cortical memory structures. Behavioral, anatomical, and physiological evidence across mammalian species has shown that interactions between the anterior thalamic nuclei, cortex and hippocampal formation are vital for spatial memory processing. Furthermore, the adjacent laterodorsal thalamic nucleus (LD), interconnected to the retrosplenial cortex (RSC) and visual system, also contributes to spatial memory in mammals. However, how these thalamic nuclei contribute to memory still remains largely unknown. Fortunately, our understanding of the importance of the thalamus in cognitive processes is being redefined, as widespread evidence challenges the established view of the thalamus as a passive relay of sensory and subcortical information to the cortex. In this review article, we examine whether the anterior thalamic nuclei and the adjacent LD are suitable candidates for "higher-order" thalamic nuclei, as defined by the Sherman and Guillery model. Rather than simply relaying information to cortex, "higher-order" thalamic nuclei have a prominent role in cognition, as they can regulate how areas of the cortex interact with one another. These considerations along with a review of the latest research will be used to suggest future studies that will clarify the contributions that the anterior and LD have in supporting cortical functions during cognitive processes.

Keywords: anterior thalamus, entorhinal cortex, grid cells, head direction cells, hippocampus, laterodorsal thalamus, prefrontal cortex, retrosplenial cortex

\section{BACKGROUND}

In mammals, interconnected brain regions, including the medial temporal lobes, frontal and cingulate cortices and diencephalon, support the formation of new memories (Aggleton, 2014). An important feature of these extended neural networks is anatomical convergence of cortical and medial temporal lobe connections within the anterior nuclei (ATN) and the laterodorsal nuclei (LD) of the thalamus. Behavioral and physiological evidence also indicate these thalamic structures are important hubs within the memory circuitry. However, how the ATN and LD are influencing this circuitry is not yet well understood.

In humans, damage to the ATN from stroke, alcohol abuse, or neurodegenerative disorders is associated with an impaired ability to form new memories (Harding et al., 2000; 
Van der Werf et al., 2000, 2003; Carlesimo et al., 2011; Kopelman, 2015; Aggleton et al., 2016; Perry et al., 2018). Animal models with damage to the ATN are also impaired in forming new memories. For example, localized ATN lesions in non-human primates impaired new learning in an episodic-like memory task (Parker and Gaffan, 1997). Similarly, excitotoxic lesions to the ATN in rodents consistently result in severe spatial memory deficits in tasks involving allocentric navigation (Aggleton and Brown, 1999; Mitchell and Dalrymple-Alford, 2005; Aggleton and Nelson, 2015; Dalrymple-Alford et al., 2015; Perry et al., 2018; Wolff and Vann, 2019). Deficits after ATN lesions are not restricted to spatial navigation though. For example, rodents are also impaired at making biconditional discriminations, contextual memory processing, forming fixed paired associations between an object and location, and reproducing accurate temporal order memory for a list of previously presented odors (Sziklas and Petrides, 1999; Gibb et al., 2006; Wolff et al., 2006; Law and Smith, 2012; Dumont et al., 2014). The contribution of the LD to spatial memory has thus far only been explicitly examined in two studies. In one study, LD inactivation resulted in increased reference memory errors in the radial arm maze, and in the other study, excitotoxic LD lesions impaired watermaze acquisition and retention of a fixed platform location (Mizumori et al., 1994; van Groen et al., 2002). Additional causal evidence from rat studies that either combined or extended lesions in the LD with those in the ATN support its role in spatial memory (Warburton et al., 1997; Wilton et al., 2001).

The ATN and LD sit at a convergence point within a complex array of cortical and subcortical connections (Figure 1; Aggleton et al., 2010; Jankowski et al., 2013; Dalrymple-Alford et al., 2015). These include widespread, often reciprocal, links to frontal cortex, cingulate cortex, especially retrosplenial cortex (RSC), and hippocampal formation (Shibata, 1998, 2000; van Groen et al., 2002; Shibata and Naito, 2005). One of the main points of difference between the ATN and LD are the primary subcortical afferents they receive. The ATN receive their primary ascending afferents from the mammillary bodies (MB), which are also strongly implicated in mnemonic processing (Vann, 2010). The inputs to the MB originate in the vestibular system and run via the midbrain tegmental nuclei of Gudden (Guillery, 1955, 1956; Taube, 2007). The LD receives its primary ascending afferents from visual structures, including the pretectum, superior colliculus and ventral lateral geniculate nucleus (Thompson and Robertson, 1987).

The ATN can be divided into three subnuclei: anterodorsal $(\mathrm{AD})$, anteroventral $(\mathrm{AV})$, and anteromedial nuclei (AM: Figure 1). Differences in their connectivity have been tied to specific functional distinctions between them (Aggleton et al., 2010). For an excellent description of the anatomical connectivity of the ATN across species, see Bubb et al. (2017). In contrast, anatomical and functional distinctions of the LD are not as well defined, but there is some evidence for a dorsolateral-ventromedial divide (Thompson and Robertson, 1987). The known neuroanatomical connectivity indicates that the LD provides key visual inputs to the extended hippocampal system and entorhinal cortex.
The dorsal aspect of the $\mathrm{LD}$, and the $\mathrm{AD}$ are proposed to form part of a lateral head direction circuit along with the postsubiculum, lateral MB, and RSC (Taube, 2007). This circuit is characterized by cells that preferentially fire when the animals' head is oriented in a specific direction, acting somewhat like a compass. Recent evidence indicates that head direction cells in both the $\mathrm{LD}$ and $\mathrm{AD}$ coded separately the rat's heading and movement directions (Enkhjargal et al., 2014). Head direction cells in the LD have been reported to differ from those in the $\mathrm{AD}$, in that they are highly dependent on visual cues, whereas head direction cells in the $\mathrm{AD}$ can form highly directional firing after initial exposure to an environment, and can be maintained in the absence of visual cues (Mizumori and Williams, 1993; Goodridge et al., 1998). These differences are likely generated from differences in their respective inputs (Figure 1). The functional implication of these differences is not yet clear, although both types of information are clearly necessary for effective navigation.

In contrast to the $\mathrm{LD}$ and $\mathrm{AD}$, the $\mathrm{AV}$ and $\mathrm{AM}$ are proposed to form part of a theta circuit with the medial MB, prefrontal cortex (PFC), RSC, and hippocampal formation (Vann and Aggleton, 2004; Jankowski et al., 2013). Theta rhythms (3-8 Hz in humans but $4-12 \mathrm{~Hz}$ in rodents) within this circuitry are thought to synchronize distally located populations of neurons and provide a framework for the inter-structural communication necessary for complex cognitive functions, such as memory processing (Buzsáki, 2002, 2005; Kirk and Mackay, 2003; Rutishauser et al., 2010; Colgin, 2011). The AV and AM also contain some head direction cells, and the AM some grid-like cells, suggesting they may be important convergence points between multiple streams of information that are filtered and passed on to cortex (Aggleton et al., 2010; Tsanov et al., 2011a,b,c; Jankowski et al., 2015).

Important differences between the $\mathrm{LD}, \mathrm{AV}, \mathrm{AD}$ and $\mathrm{AM}$ are also observed in the pattern of cortical connections they receive (Figure 1). This is especially true for the AM, which is linked to many areas of PFC, including medial PFC and anterior cingulate cortex (ACg; Hoover and Vertes, 2007; Xiao et al., 2009; Jankowski et al., 2013). Further differences are found in their respective links with RSC. The AD and AV are predominantly interconnected with granular RSC, which is principally involved in navigational processing, while the AM is predominantly connected with dysgranular RSC, which is principally involved in visual processing (van Groen and Wyss, 1990, 1992, 1995, 2003; van Groen et al., 1999; Shibata, 1998). The LD has reciprocal connections with both granular and dysgranular RSC (Sripanidkulchai and Wyss, 1986; Shibata, 1998, 2000). Further, RSC afferents to the AD, AV and AM originated in layer VI, suggesting that RSC modulates how ATN communicate with other structures, whereas LD receives both layer $\mathrm{V}$ (driver) and VI (modulator) inputs. The LD also has reciprocal projections with Brodmann area $18 \mathrm{~b}$ of the visual cortex, whereas AM only projects to visual cortex (Thompson and Robertson, 1987; van Groen and Wyss, 1992; Shibata and Naito, 2005). Finally, only LD and AV share reciprocal connections with secondary motor cortex, but all four thalamic nuclei project to entorhinal cortex (Shibata and Naito, 2005). 
A

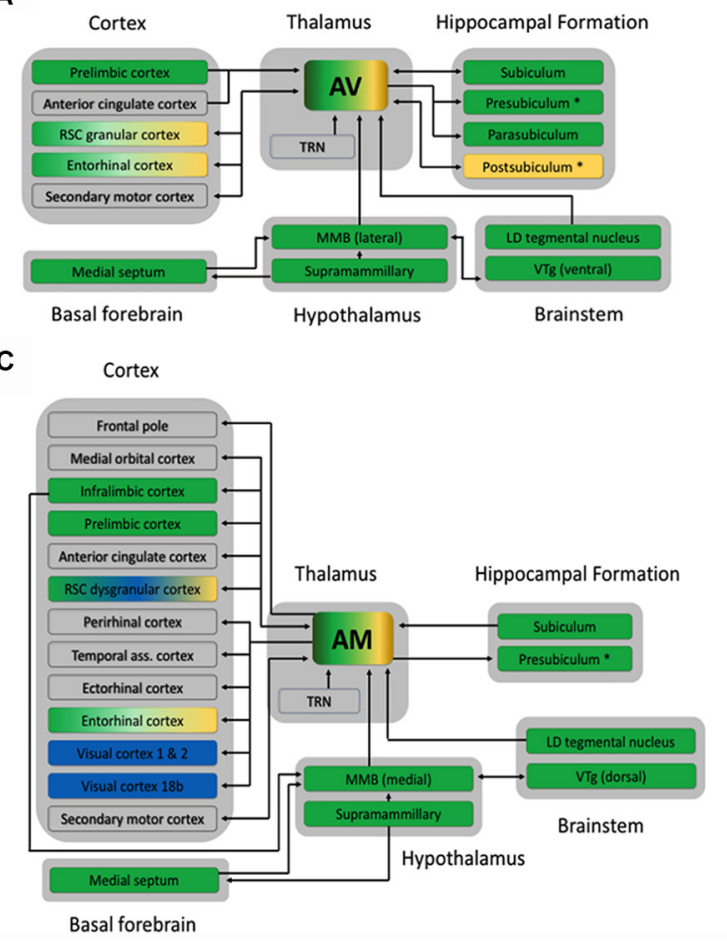

B

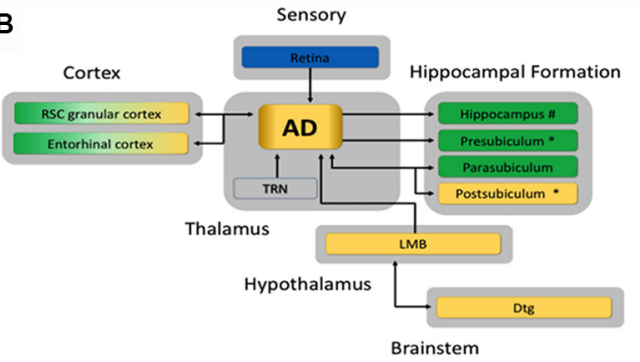

D

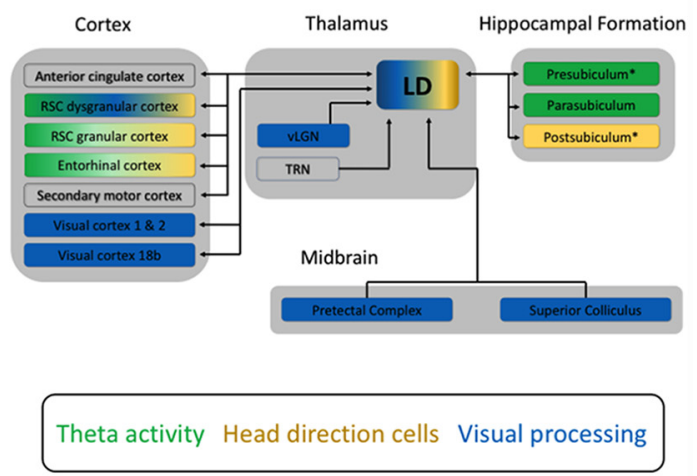

FIGURE 1 | Schematic diagrams outlining the main connections of (A) the anteroventral (AV), (B) the anterodorsal (AD), (C) the anteromedial (AM) subnuclei of the anterior thalamic nucleus, and (D) the laterodorsal (LD) thalamic nucleus from studies in rats, cats and monkeys. All four nuclei share dense reciprocal connections to both the RSC and the hippocampal formation. Clear functionally relevant differences are apparent however, between the additional connections of each subnucleus. For example, the AM is broadly connected to many cortical sites including prefrontal, temporal and sensory cortex, whereas the AD has few cortical connections, and does not project to the anterior cingulate like the AM, AV and LD. Another critical point of difference is that all three subnuclei of the ATN receive one primary input containing mnemonically relevant information from the mammillary bodies (MB), whereas the LD receives ascending afferents from regions associated with visual processing, such as the pretectal complex. Arrowheads indicate the direction of information flow, with double headed arrows showing reciprocal connections between structures. The colored boxes indicate the three major functional processes, theta rhythm (green), head direction (gold) or visual processing (blue), associated with these four thalamocortical circuits. Structures associated with two or more of these processes are indicated by a combination of colors. The larger gray boxes group each structure into the broader category of brain region it belongs to, e.g., cortex. Additional connections also exist between cortical structures, the hippocampal formation, midbrain, and brainstem but these are not depicted here. We have also included the presubiculum and postsubiculum as separate structures but we note that the dorsal part of the presubiculum is commonly known as the postsubiculum. Additional abbreviations: Dtg, dorsal tegmental nucleus of Gudden; LD tegmental nucleus, laterodorsal tegmental nucleus; LMB, lateral mammillary bodies; MMB, medial mammillary bodies; RSC, retrosplenial cortex; TRN, thalamic reticular nucleus; vLGN, ventral part of the lateral geniculate nucleus of the thalamus; Visual cortex 18b, Brodmann area 18b; VTg ventral tegmental nucleus of Gudden.

\section{SUMMARY OF THE ESTABLISHED PRINCIPLES}

The thalamus sits at an important interface between the cortex and its numerous inputs. Every part of cortex receives a thalamic input, and with few exceptions, i.e., the olfactory input, the thalamus is the sole provider of sensory and subcortical information to cortex (Sherman, 2017). Early studies of transthalamic sensory relays suggested almost one to one replication of the primary ascending afferent signal in the thalamus. These findings led to the now entrenched view of the thalamus as a passive relay of information to cortex (Sherman, 2017). In this view, any cognitively relevant transformations of ascending sensory or subcortical information would only occur once they passed through thalamus and reached higher order processing sites in the cortex (Halassa, 2018). In their seminal article,
Sherman and Guillery (1996) challenged this simplistic view of thalamic function, suggesting instead that the thalamus contains at least two types of nuclei; "first" order nuclei of sensory or subcortical information as previously proposed, and also "higher" order nuclei that influence cortical activity by supporting the "transfer" of information from one area of cortex to another. Citing a large body of anatomical and physiological evidence on the visual pathway formed by the lateral geniculate nucleus, Sherman and Guillery (1996) demonstrated that even in first order nuclei, the role of the thalamus is highly dynamic with the ability to modulate the information it passes to cortex.

\section{First Order Nuclei}

"First" order thalamic nuclei are those that receive primary ascending afferents or "driver" inputs from peripheral sensory, 

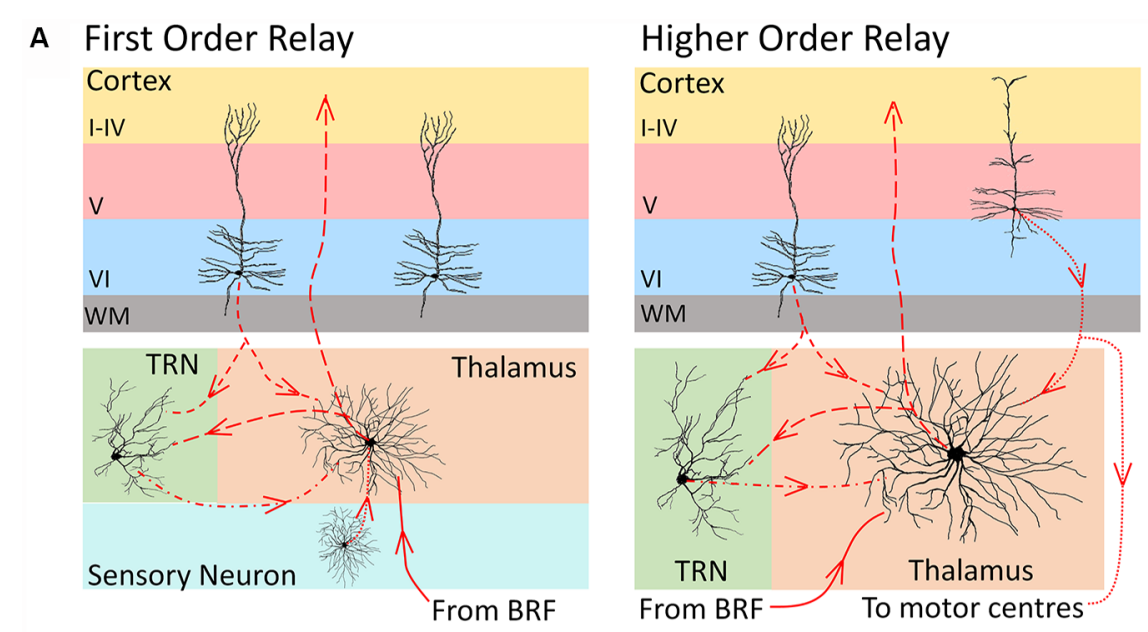

B

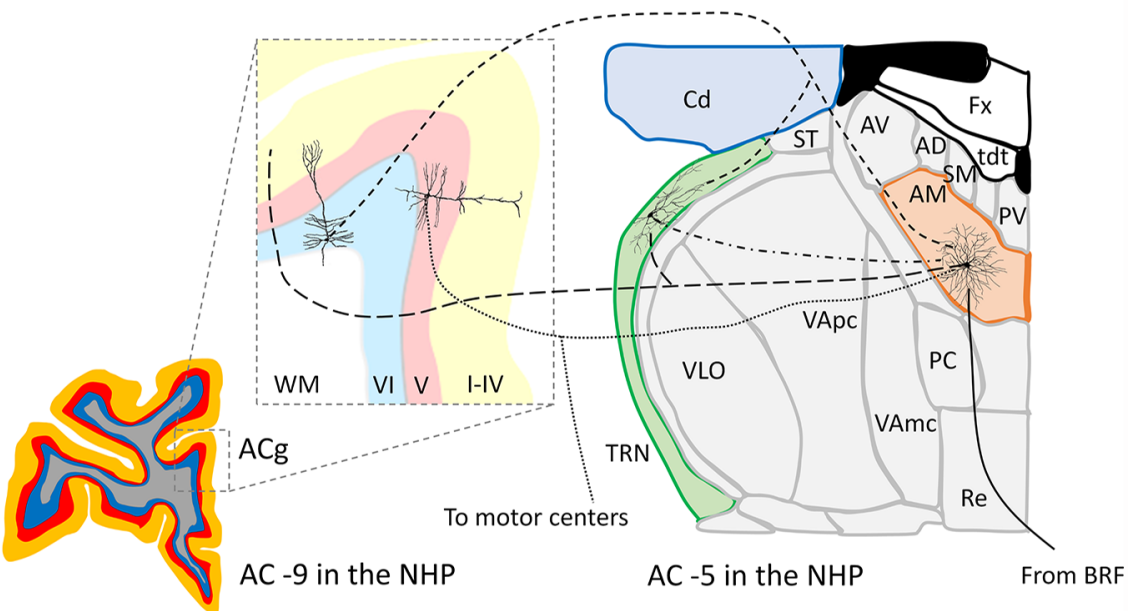

c

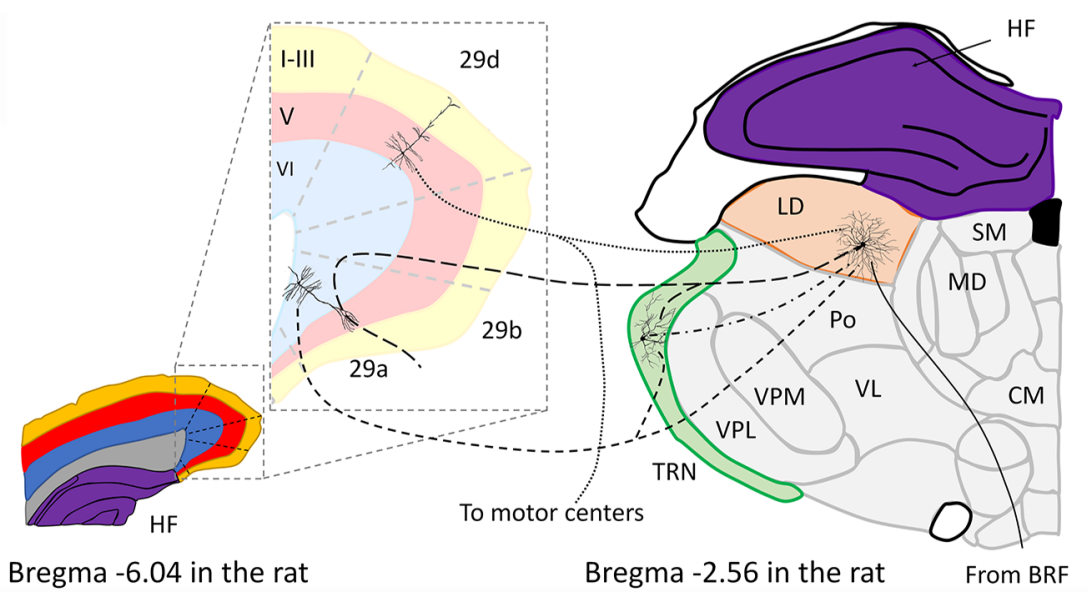

FIGURE 2 | Schematic representation (A) of the organization of a first order (left panel) and higher order (right panel) thalamic relay according to the Sherman and Guillery (1996) model. Panel (B) depicts a hypothetical scenario based on the work of Xiao and Barbas (2002) and Xiao et al. (2009) of the anteromedial subnucleus (AM, orange) of the anterior thalamic nuclei as a higher order thalamic relay to anterior cingulate cortex (ACg) in the macaque monkey. Panel (C) depicts a hypothetical scenario based on the work of Shibata (2000) and Thompson and Robertson (1987) of the laterodorsal thalamic nucleus (LD, orange) as a higher order relay to the dysgranular (29d) retrosplenial cortex in a rat (Shibata, 2000). In a higher order thalamic relay both a "driver" afferent from layer $V$ of the cortex (dotted lines) and a "modulator" afferent from layer VI of cortex (short dashed lines) and the (TRN, green) innervates the thalamic relay neuron. The thalamic relay neuron then in turn projects this cortical information back to layers of cortex (large dashed lines). Projections from the brainstem reticular formation (BRF) and directly from the TRN provide additional modulation to these thalamic relay neurons (Sherman, 2017). Coronal sections for the macaque monkey (B) adapted from http://braininfo.rprc. washington.edu/PrimateBrainMaps/atlas/Mapcorindex.html. Images taken at $-9 \mathrm{~mm}$ and $-5 \mathrm{~mm}$ from the $\mathrm{AC}$ in the macaque brain. Coronal sections for the rat

(Continued) 
FIGURE 2 | Continued

(C) adapted from Paxinos and Watson (1998). Images taken -6.04 mm and $-2.56 \mathrm{~mm}$ from Bregma in the rat brain. Additional abbreviations: 29a-b, Brodmann area 29a-b, granular retrosplenial cortex; 29d, Brodmann area 29d, dysgranular retrosplenial cortex; AC, anterior commissure; AD, anterodorsal subnucleus of the anterior thalamic nuclei; $\mathrm{AV}$, anteroventral subnucleus of the anterior thalamic nuclei; $\mathrm{Fx}$, fornix; $\mathrm{Cd}$, caudate nucleus; $\mathrm{CM}$, centromedial nucleus of the thalamus; $\mathrm{HF}$, hippocampal formation; MD, mediodorsal thalamus; PC, paracentral nucleus; Po, posterior thalamic group; PV, paraventricular nucleus; Re, nucleus reuniens of the thalamus; SM, stria medullaris; ST, stria terminalis; tdt, telodiencephalic fissure; VApc, ventroanterior nucleus (parvicellular); VAmc, ventroanterior nucleus (magnocellular); VI, Layer six of cortex; V, Layer five of cortex; I-IV, Layers one to four of cortex; VL, ventrolateral thalamus; VLO, oral part of the ventrolateral nucleus; VPL, ventroposterolateral thalamus; VPM, ventroposteromedial thalamus, WM, white matter.

or subcortical regions (Sherman and Guillery, 1996). One example is the retinal input into the lateral geniculate nucleus of the thalamus, which is "relayed" to visual cortex. First order nuclei also receive distinct fine "modulator" afferents from layer VI of the cortex (Sherman and Guillery, 1996; Sherman, 2016). This modulation is generally linked to the inhibitory GABA pathway passing through the thalamic reticular nucleus (TRN). Modulator inputs form part of a reciprocal circuit, meaning that the layer VI cortical afferents project to the same thalamic region that innervates the layer VI cortical neurons (Sherman, 2016). The "driver" inputs provide the major functional input to the thalamic relay cells and the "modulator" cortico-reticular-thalamic inputs provide a means to "gate" or control the flow of information to cortex (Sherman, 2016).

\section{Higher Order Nuclei}

Unlike "first" order nuclei, "higher" order nuclei receive few or no comparable ascending sensory or subcortical afferents but instead receive two types of afferents from cortex (Sherman and Guillery, 1996). One of these is just like the layer VI modulatory cortico-reticular-thalamic input received by first order nuclei. The other is comprised of coarse afferents from pyramidal cells located in layer V (Sherman, 2016). Therefore, higher order nuclei represent part of a feed-forward cortico-thalamocortical pathway that "relays" information from one part of the cortex to another. Interestingly, recent evidence has shown that optogenetic activation of the mediodorsal thalamic nucleus (MD), a higher order nucleus for PFC, does not appear to alter the specificity of cortical representations, but rather enhances the local effective connectivity within the PFC (Schmitt et al., 2017).

Given the prominence of the ATN and LD in memory formation, it is worth considering how they might fit the Sherman and Guillery (1996) model. Such a consideration drives a number of testable hypotheses regarding the functional contribution of ATN and LD to the wider extended hippocampal memory circuit and perhaps could further our understanding of why such profound memory deficits occur when they are damaged. The next section examines the state of our current knowledge with regards to the functional interactions between ATN, LD and their interconnected cortical sites.

\section{CURRENT STATE OF THE ART}

The known neuroanatomical differences indicate that rather than considering either the ATN or LD as a whole structure, we should instead consider their subnuclei as separate entities. Previous work has shown that the physiological attributes of the driving inputs to the $\mathrm{AD}$ from the lateral $\mathrm{MB}$, and modulatory afferents from cortex implicate it as a first order relay (Petrof and Sherman, 2009). Further, novel molecular evidence has reinforced the functional heterogeneity of ATN subnuclei. Phillips et al. (2018) developed a comprehensive transcriptomic atlas of mouse thalamus. The majority of thalamic nuclei belong to one of three major clusters, which appear to lie on a single continuum relating to the thalamic mediodorsal axis, with any given cortical region getting input from each of these clusters. Interestingly, ATN subnuclei did not cluster together, rather AV along with LD fell into the "primary" cluster. Nuclei within this cluster were enriched in gene encoding neurotransmitters, ion channels, and signaling molecules, all of which contribute to faster channel kinetics and narrower action potentials. By contrast, AM, along with regions like MD, fell into the "secondary" cluster, which were strongly enriched in neuromodulatory genes. There is strong evidence that at least one subnucleus of $\mathrm{MD}$, the parvocellular $\mathrm{MD}$ in non-human primates, is a higher order relay for dorsolateral PFC, as it receives inputs from both layer V and VI neurons and appears to modulate intercortical connectivity (Schwartz et al., 1991; Rovó et al., 2012; Mitchell, 2015; Collins et al., 2018). The AM also appears to receive inputs from layer V and VI of the cortex, at least in non-human primates, raising the possibility that it may act as a higher order relay (Xiao et al., 2009), although in rat, it has been categorized as a first order relay (Varela, 2014). Interestingly, the mouse $\mathrm{AD}$ did not appear to conform to any of the three clusters defined by Phillips et al. (2018).

Further to these molecular differences, there is growing evidence that ATN is more than a passive relay of hypothalamic and brainstem information to cortex. Recent work has shown how selective manipulations in ATN have a profound impact across many structures in the limbic cortex, likely contributing to the cognitive deficits observed in mammals with ATN damage. For example, temporary inactivation of rat ATN altered grid-like firing patterns of medial entorhinal cortex (MEC) neurons, while ATN lesions reduced the number of grid-cell neurons in the MEC (Winter et al., 2015). This evidence supports the hypothesis that head direction cell inputs from ATN are involved in the formation of MEC grid cell patterns (Winter et al., 2015). Further, viral tracers demonstrated the pathway for head direction information transfer from the AD onto MEC via the presubiculum (Huang et al., 2017), with the inhibitory micro-circuity within presubiculum possibly maintaining the head direction signal (Simonnet et al., 2017; Simonnet and Fricker, 2018). In addition, ATN lesions in rats also result in microstructural changes in the hippocampus and RSC (Harland et al., 2014). Along with severe spatial memory impairments, Harland et al. (2014) observed substantial reductions in dendritic spine densities, which are associated with synaptic plasticity in hippocampal CA1 and RSC granular b cortex. Finally, high- 
frequency stimulation $(\sim 130 \mathrm{~Hz})$ of rodent ATN increased neurogenesis in the dentate gyrus and aided performance on memory tasks (Toda et al., 2008; Encinas et al., 2011; Hamani et al., 2011).

Similarly, stimulation of ATN in larger mammals modulated hippocampal field potential in a frequency dependent manner and increased the BOLD response in hippocampus and PFC (Stypulkowski et al., 2014; Gibson et al., 2016); and finally in humans, recordings from multiple depth electrodes in patients with epilepsy showed high-frequency stimulation $(\sim 130 \mathrm{~Hz})$ of ATN was capable of decoupling large scale neural networks that included hippocampus, insular cortex, parahippocampal cortex and dorsolateral PFC (Yu et al., 2018).

\section{FUTURE DIRECTIONS}

Our understanding of cortico-thalamo-cortical interactions and their purpose are still limited, especially with respect to higher order relays. However, based on current findings, there appears to be some evidence that AM (Figure 2) might act as a higher order relay to cortex in primates, while the $\mathrm{AD}$ is a first order relay. However, how AV and subnuclei of the LD influence cortex still remain to be fully explored. Influences from outside the limbic circuitry also need to be investigated. For example, inputs from dorsal striatum and medial precentral cortex are likely to be modulating theta within ATN, LD, hippocampal formation and MEC for grid cell formation (Mehlman et al., 2019a,b). Also key to our understanding is whether the relationship of any cortico-thalamo-cortical projections involving ATN and LD are conserved across species. Thus, far cortico-thalamocortical interactions have focused heavily on rodent models (Sherman, 2016; Schmitt et al., 2017). Mice and rats provide a great starting point for proof of principle, but they lack the cortical and thalamic development present in higher order species, such as non-human primates and humans (Halassa, 2018). Thus, it is likely that there are differences in fundamental aspects of thalamocortical circuits across species still waiting to be discovered.

What still remains to be understood in neuroscience, and with specific relevance to this review article, is how ATN and LD are managing the various streams of afferent information they receive; clearly the layer VI projections from the RSC are important (Mitchell et al., 2018). Furthermore, it is critical that the nature of the efferent signals they pass on to cortex is characterized. Animal and human experiments that record neural activity from ATN and LD subnuclei and their cortical targets during relevant behavioral tasks will be of great

\section{REFERENCES}

Aggleton, J. P. (2014). Looking beyond the hippocampus: old and new neurological targets for understanding memory disorders. Proc. Biol. Sci. 281:20140565. doi: 10.1098/rspb.2014.0565

Aggleton, J. P., and Brown, M. W. (1999). Episodic memory, amnesia, and the hippocampal-anterior thalamic axis. Behav. Brain Sci. 22, 425-444; discussion 444-489. doi: 10.1017/s0140525x99002034 interest. Altering thalamic, striatum, or cortical functioning, using pharmacological agents or optogenetics, and targeting specific cell layers or cell types using transgenic, or viral vector approaches will also be essential to dissecting the specific learning and memory, and navigational functions of these thalamocortical circuits.

Finally, imaging techniques are still constrained by a lack of resolution and continue to struggle to define individual thalamic nuclei (Aggleton et al., 2016). However, using a 7T magnetic imaging scanner and advanced image processing techniques, some of the microstructural components of the MD could be elucidated in humans (Pergola et al., 2018). Consequently, similar strategies may be applied to cognitive and behavioral neuroscience studies investigating ATN and $\mathrm{LD}$, with the caveat that for the ATN at least, it is a much smaller thalamic structure. There has also been increasing work examining ATN-cortical interactions during electrode implant surgeries for refractory epilepsy in humans. We hope that such opportunities will be utilized more in the future, especially in conjunction with detailed cognitive and behavioral tasks and advanced neuroimaging analyses of these patients.

\section{CONCLUDING REMARKS}

Evidence from animals and humans support the importance of cortical and subcortical interactions during cognitive processes, including learning and memory, and navigation. Modern neuroscience techniques must now be used to explore how and why these interactions are so critical when we are learning new information, or optimizing our behaviors. In order to advance our knowledge, we must characterize the underlying mechanisms that support these interactions between neural structures important for forming new memories, both in the normal brain, for which animal models remain essential, and in patients with neurodegenerative diseases and neuropsychiatric disorders.

\section{AUTHOR CONTRIBUTIONS}

$\mathrm{BP}$ and AM both contributed to the writing of this manuscript.

\section{FUNDING}

The authors are supported by a Wellcome Trust Senior Fellowship to AM (110157/Z/15/Z). This article is published with open access funds from the COAF Block Grant to the University of Oxford. thalamic nuclei cause such severe spatial deficits? Neurosci. Biobehav. Rev. 54, 131-144. doi: 10.1016/j.neubiorev.2014.08.013

Aggleton, J. P., O’Mara, S. M., Vann, S. D., Wright, N. F., Tsanov, M., and Erichsen, J. T. (2010). Hippocampal-anterior thalamic pathways for memory: uncovering a network of direct and indirect actions. Eur. J. Neurosci. 31, 2292-2307. doi: 10.1111/j.1460-9568.2010. 07251.x 
Aggleton, J. P., Pralus, A., Nelson, A. J., and Hornberger, M. (2016). Thalamic pathology and memory loss in early Alzheimer's disease: moving the focus from the medial temporal lobe to Papez circuit. Brain 139, 1877-1890. doi: 10.1093/brain/aww083

Bubb, E. J., Kinnavane, L., and Aggleton, J. P. (2017). Hippocampaldiencephalic_cingulate networks for memory and emotion: an anatomical guide. Brain Neurosci. Adv. 1:2398212817723443. doi: 10.1177/ 2398212817723443

Buzsáki, G. (2002). Theta oscillations in the hippocampus. Neuron 33, 325-340. doi: 10.1016/s0896-6273(02)00586-x

Buzsáki, G. (2005). Theta rhythm of navigation: link between path integration and landmark navigation, episodic and semantic memory. Hippocampus 15, 827-840. doi: 10.1002/hipo.20113

Carlesimo, G. A., Lombardi, M. G., and Caltagirone, C. (2011). Vascular thalamic amnesia: a reappraisal. Neuropsychologia 49, 777-789. doi: 10.1016/j. neuropsychologia.2011.01.026

Colgin, L. L. (2011). Oscillations and hippocampal-prefrontal synchrony. Curr. Opin. Neurobiol. 21, 467-474. doi: 10.1016/j.conb.2011.04.006

Collins, D. P., Anastasiades, P. G., Marlin, J. J., and Carter, A. G. (2018). Reciprocal circuits linking the prefrontal cortex with dorsal and ventral thalamic nuclei. Neuron 98, 366.e4-379.e4. doi: 10.1016/j.neuron.2018.03.024

Dalrymple-Alford, J. C., Harland, B., Loukavenko, E. A., Perry, B., Mercer, S., Collings, D. A., et al. (2015). Anterior thalamic nuclei lesions and recovery of function: relevance to cognitive thalamus. Neurosci. Biobehav. Rev. 54, 145-160. doi: 10.1016/j.neubiorev.2014.12.007

Dumont, J. R., Wright, N. F., Pearce, J. M., and Aggleton, J. P. (2014). The impact of anterior thalamic lesions on active and passive spatial learning in stimulus controlled environments: geometric cues and pattern arrangement. Behav. Neurosci. 128, 161-177. doi: 10.1037/a0036280

Encinas, J. M., Hamani, C., Lozano, A. M., and Enikolopov, G. (2011). Neurogenic hippocampal targets of deep brain stimulation. J. Comp. Neurol. 519, 6-20. doi: $10.1002 / \mathrm{cne} .22503$

Enkhjargal, N., Matsumoto, J., Chinzorig, C., Berthoz, A., Ono, T., and Nishijo, H. (2014). Rat thalamic neurons encode complex combinations of heading and movement directions and the trajectory route during translocation with sensory conflict. Front. Behav. Neurosci. 8:242. doi: 10.3389/fnbeh. 2014.00242

Gibb, S. J., Wolff, M., and Dalrymple-Alford, J. C. (2006). Odour-place pairedassociate learning and limbic thalamus: comparison of anterior, lateral and medial thalamic lesions. Behav. Brain Res. 172, 155-168. doi: 10.1016/j.bbr. 2006.05.017

Gibson, W. S., Ross, E. K., Han, S. R., Van Gompel, J. J., Min, H. K., and Lee, K. H. (2016). Anterior thalamic deep brain stimulation: functional activation patterns in a large animal model. Brain Stimul. 9, 770-773. doi: 10.1016/j.brs.2016. 04.012

Goodridge, J. P., Dudchenko, P. A., Worboys, K. A., Golob, E. J., and Taube, J. S. (1998). Cue control and head direction cells. Behav. Neurosci. 112, 749-761. doi: 10.1037/0735-7044.112.4.749

Guillery, R. W. (1955). A quantitative study of the mamillary bodies and their connexions. J. Anat. 89, 19-32.

Guillery, R. W. (1956). Degeneration in the post-commissural fornix and the mamillary peduncle of the rat. J. Anat. 90, 350-370.

Halassa, M. M. (2018). Fronto-thalamic architectures for cognitive algorithms. Neuron. 98, 237-239. doi: 10.1016/j.neuron.2018.04.006

Hamani, C., Stone, S. S., Garten, A., Lozano, A. M., and Winocur, G. (2011). Memory rescue and enhanced neurogenesis following electrical stimulation of the anterior thalamus in rats treated with corticosterone. Exp. Neurol. 232, 100-104. doi: 10.1016/j.expneurol.2011.08.023

Harding, A., Halliday, G., Caine, D., and Kril, J. (2000). Degeneration of anterior thalamic nuclei differentiates alcoholics with amnesia. Brain. 123, 141-154. doi: 10.1093/brain/123.1.141

Harland, B. C., Collings, D. A., McNaughton, N., Abraham, W. C., and DalrympleAlford, J. C. (2014). Anterior thalamic lesions reduce spine density in both hippocampal CA1 and retrosplenial cortex, but enrichment rescues CA1 spines only. Hippocampus 24, 1232-1247. doi: 10.1002/hipo.22309

Hoover, W. B., and Vertes, R. P. (2007). Anatomical analysis of afferent projections to the medial prefrontal cortex in the rat. Brain Struct. Funct. 212, 149-179. doi: 10.1007/s00429-007-0150-4
Huang, L. W., Simonnet, J., Nassar, M., Richevaux, L., Lofredi, R., and Fricker, D. (2017). Laminar Localization and projection-specific properties of presubicular neurons targeting the lateral mammillary nucleus, thalamus, or medial entorhinal cortex. Heliyon 4:ENEURO.0370-16.2017. doi: 10.1523/eneuro. 0370-16.2017

Jankowski, M. M., Passecker, J., Islam, M. N., Vann, S., Erichsen, J. T., Aggleton, J. P., et al. (2015). Evidence for spatially-responsive neurons in the rostral thalamus. Front. Behav. Neurosci. 9:256. doi: 10.3389/fnbeh. 2015.00256

Jankowski, M. M., Ronnqvist, K. C., Tsanov, M., Vann, S. D., Wright, N. F., Erichsen, J. T., et al. (2013). The anterior thalamus provides a subcortical circuit supporting memory and spatial navigation. Front. Syst. Neurosci. 7:45. doi: 10.3389/fnsys.2013.00045

Kirk, I. J., and Mackay, J. C. (2003). The role of theta-range oscillations in synchronising and integrating activity in distributed mnemonic networks. Cortex 39, 993-1008. doi: 10.1016/s0010-9452(08)70874-8

Kopelman, M. D. (2015). What does a comparison of the alcoholic Korsakoff syndrome and thalamic infarction tell us about thalamic amnesia? Neurosci. Biobehav. Rev. 54, 46-56. doi: 10.1016/j.neubiorev.2014.08.014

Law, L. M., and Smith, D. M. (2012). The anterior thalamus is critical for overcoming interference in a context-dependent odor discrimination task. Behav. Neurosci. 126, 710-719. doi: 10.1037/a0029698

Mehlman, M. L., Winter, S. S., and Taube, J. S. (2019a). Functional and anatomical relationships between the medial precentral cortex, dorsal striatum, and head direction cell circuitry. II. Neuroanatomical studies. J. Neurophysiol. 121, 371-395. doi: 10.1152/jn.00144.2018

Mehlman, M. L., Winter, S. S., Valerio, S., and Taube, J. S. (2019b). Functional and anatomical relationships between the medial precentral cortex, dorsal striatum, and head direction cell circuitry. J. Neurophysiol. 121, 350-370. doi: 10.1152/jn. 00143.2018

Mitchell, A. S. (2015). The mediodorsal thalamus as a higher order thalamic relay nucleus important for learning and decision-making. Neurosci. Biobehav. Rev. 54, 76-88. doi: 10.1016/j.neubiorev.2015.03.001

Mitchell, A. S., Czajkowski, R., Zhang, N., Jeffery, K., and Nelson, A. (2018). Retrosplenial cortex and its role in spatial cognition. Brain Neurosci. Adv. 2:2398212818757098. doi: 10.1177/2398212818757098

Mitchell, A. S., and Dalrymple-Alford, J. C. (2005). Dissociable memory effects after medial thalamus lesions in the rat. Eur. J. Neurosci. 22, 973-985. doi: 10.1111/j.1460-9568.2005.04199.x

Mizumori, S. J., Miya, D. Y., and Ward, K. E. (1994). Reversible inactivation of the lateral dorsal thalamus disrupts hippocampal place representation and impairs spatial learning. Brain Res. 644, 168-174. doi: 10.1016/0006-8993(94)90361-1

Mizumori, S. J., and Williams, J. D. (1993). Directionally selective mnemonic properties of neurons in the lateral dorsal nucleus of the thalamus of rats. J. Neurosci. 13, 4015-4028. doi: 10.1523/JNEUROSCI.13-09-04015.1993

Parker, A., and Gaffan, D. (1997). The effect of anterior thalamic and cingulate cortex lesions on object-in-place memory in monkeys. Neuropsychologia 35, 1093-1102. doi: 10.1016/s0028-3932(97)00042-0

Paxinos, G., and Watson, C. (1998). The Rat Brain in Stereotaxic Coordinates. 4th Edn. New York, NY: Academic Press.

Pergola, G., Danet, L., Pitel, A. L., Carlesimo, G. A., Segobin, S., Pariente, J., et al. (2018). The regulatory role of the human mediodorsal thalamus. Trends Cogn. Sci. 22, 1011-1025. doi: 10.1016/j.tics.2018.08.006

Perry, B. A. L., Mercer, S. A., Barnett, S. C., Lee, J., and DalrympleAlford, J. C. (2018). Anterior thalamic nuclei lesions have a greater impact than mammillothalamic tract lesions on the extended hippocampal system. Hippocampus 28, 121-135. doi: 10.1002/hipo.22815

Petrof, I., and Sherman, S. M. (2009). Synaptic properties of the mammillary and cortical afferents to the anterodorsal thalamic nucleus in the mouse. J. Neurosci. 29, 7815-7819. doi: 10.1523/JNEUROSCI.1564-09.2009

Phillips, J. W., Schulmann, A., Hara, E., Liu, C., Wang, L., Shields, B., et al. (2018). A single spectrum of neuronal identities across thalamus. bioXriv [Preprint] doi: $10.1101 / 241315$

Rovó, Z., Ulbert, I., and Acsády, L. (2012). Drivers of the primate thalamus. J. Neurosci. 32, 17894-17908. doi: 10.1523/JNEUROSCI.2815-12.2012

Rutishauser, U., Ross, I. B., Mamelak, A. N., and Schuman, E. M. (2010). Human memory strength is predicted by theta-frequency phase-locking of single neurons. Nature 464, 903-907. doi: 10.1038/nature08860 
Schmitt, L. I., Wimmer, R. D., Nakajima, M., Happ, M., Mofakham, S., and Halassa, M. M. (2017). Thalamic amplification of cortical connectivity sustains attentional control. Nature 545, 219-223. doi: 10.1038/nature22073

Schwartz, M. L., Dekker, J. J., and Goldman-Rakic, P. S. (1991). Dual mode of corticothalamic synaptic termination in the mediodorsal nucleus of the rhesus monkey. J. Comp. Neurol. 309, 289-304. doi: 10.1002/cne.903090302

Sherman, S. M. (2016). Thalamus plays a central role in ongoing cortical functioning. Nat. Neurosci. 19, 533-541. doi: 10.1038/nn.4269

Sherman, S. M. (2017). Functioning of circuits connecting thalamus and cortex. Compr. Physiol. 7, 713-739. doi: 10.1002/cphy.c160032

Sherman, S. M., and Guillery, R. W. (1996). Functional organization of thalamocortical relays. J. Neurophysiol. 76, 1367-1395. doi: 10.1152/jn.1996.76. 3.1367

Shibata, H. (1998). Organization of projections of rat retrosplenial cortex to the anterior thalamic nuclei. Eur. J. Neurosci. 10, 3210-3219. doi: 10.1046/j.14609568.1998.00328.x

Shibata, H. (2000). Organization of retrosplenial cortical projections to the laterodorsal thalamic nucleus in the rat. Neurosci. Res. 38, 303-311. doi: 10.1016/s0168-0102(00)00174-7

Shibata, H., and Naito, J. (2005). Organization of anterior cingulate and frontal cortical projections to the anterior and laterodorsal thalamic nuclei in the rat. Brain Res. 1059, 93-103. doi: 10.1016/j.brainres.2005.08.025

Simonnet, J., and Fricker, D. (2018). Cellular components and circuitry of the presubiculum and its functional role in the head direction system. Cell Tissue Res. 373, 541-556. doi: 10.1007/s00441-018-2841-y

Simonnet, J., Nassar, M., Stella, F., Cohen, I., Mathon, B., Boccara, C. N., et al. (2017). Activity dependent feedback inhibition may maintain head direction signals in mouse presubiculum. Nat. Commun. 8:16032. doi: $10.1038 /$ ncomms 16032

Sripanidkulchai, K., and Wyss, J. M. (1986). Thalamic projections to retrosplenial cortex in the rat. J. Comp. Neurol. 254, 143-165. doi: 10.1002/cne.902540202

Stypulkowski, P. H., Stanslaski, S. R., Jensen, R. M., Denison, T. J., and Giftakis, J. E. (2014). Brain stimulation for epilepsy-local and remote modulation of network excitability. Brain Stimul. 7, 350-358. doi: 10.1016/j. brs.2014.02.002

Sziklas, V., and Petrides, M. (1999). The effects of lesions to the anterior thalamic nuclei on object-place associations in rats. Eur. J. Neurosci. 11, 559-566. doi: 10.1046/j.1460-9568.1999.00448.x

Taube, J. S. (2007). The head direction signal: origins and sensory-motor integration. Annu. Rev. Neurosci. 30, 181-207. doi: 10.1146/annurev.neuro.29. 051605.112854

Thompson, S. M., and Robertson, R. T. (1987). Organization of subcortical pathways for sensory projections to the limbic cortex. II. Afferent projections to the thalamic lateral dorsal nucleus in the rat. J. Comp. Neurol. 265, 189-202. doi: 10.1002/cne.902650203

Toda, H., Hamani, C., Fawcett, A. P., Hutchison, W. D., and Lozano, A. M. (2008). The regulation of adult rodent hippocampal neurogenesis by deep brain stimulation. J. Neurosurg. 108, 132-138. doi: 10.3171/jns/2008/108/01/0132

Tsanov, M., Chah, E., Vann, S. D., Reilly, R. B., Erichsen, J. T., Aggleton, J. P., et al. (2011a). Theta-modulated head direction cells in the rat anterior thalamus. J. Neurosci. 31, 9489-9502. doi: 10.1523/JNEUROSCI.0353-11.2011

Tsanov, M., Chah, E., Wright, N., Vann, S. D., Reilly, R., Erichsen, J. T., et al. (2011b). Oscillatory entrainment of thalamic neurons by theta rhythm in freely moving rats. J. Neurophysiol. 105, 4-17. doi: 10.1152/jn.00771.2010

Tsanov, M., Wright, N., Vann, S. D., Erichsen, J. T., Aggleton, J. P., and O'Mara, S. M. (2011c). Hippocampal inputs mediate theta-related plasticity in anterior thalamus. Neuroscience 187, 52-62. doi: 10.1016/j.neuroscience.2011. 03.055

Van der Werf, Y. D., Scheltens, P., Lindeboom, J., Witter, M. P., Uylings, H. B., and Jolles, J. (2003). Deficits of memory, executive functioning and attention following infarction in the thalamus; a study of 22 cases with localised lesions. Neuropsychologia 41, 1330-1344. doi: 10.1016/s0028-3932(03)00059-9

Van der Werf, Y. D., Witter, M. P., Uylings, H. B., and Jolles, J. (2000). Neuropsychology of infarctions in the thalamus: a review. Neuropsychologia 38, 613-627. doi: 10.1016/s0028-3932(99)00104-9 van Groen, T., Kadish, I., and Wyss, J. M. (1999). Efferent connections of the anteromedial nucleus of the thalamus of the rat. Brain Res. Rev. 30, 1-26. doi: 10.1016/s0165-0173(99)00006-5

van Groen, T., Kadish, I., and Wyss, J. M. (2002). The role of the laterodorsal nucleus of the thalamus in spatial learning and memory in the rat. Behav. Brain Res. 136, 329-337. doi: 10.1016/s0166-4328(02)00199-7

van Groen, T., and Wyss, J. M. (1990). Connections of the retrosplenial granular a cortex in the rat. J. Comp. Neurol. 300, 593-606. doi: 10.1002/cne. 903000412

van Groen, T., and Wyss, J. M. (1992). Projections from the laterodorsal nucleus of the thalamus to the limbic and visual cortices in the rat. J. Comp. Neurol. 324, 427-448. doi: 10.1002/cne.903240310

van Groen, T., and Wyss, J. M. (1995). Projections from the anterodorsal and anteroventral nucleus of the thalamus to the limbic cortex in the rat. J. Comp. Neurol. 358, 584-604. doi: 10.1002/cne.903580411

van Groen, T., and Wyss, J. M. (2003). Connections of the retrosplenial granular b cortex in the rat. J. Comp. Neurol. 463, 249-263. doi: 10.1002/cne. 10757

Vann, S. D. (2010). Re-evaluating the role of the mammillary bodies in memory. Neuropsychologia 48, 2316-2327. doi: 10.1016/j.neuropsychologia. 2009.10.019

Vann, S. D., and Aggleton, J. P. (2004). The mammillary bodies: two memory systems in one? Nat. Rev. Neurosci. 5, 35-44. doi: 10.1038/nrn1299

Varela, C. (2014). Thalamic neuromodulation and its implications for executive networks. Front. Neural Circuits 8:69. doi: 10.3389/fncir.2014.00069

Warburton, E. C., Baird, A. L., and Aggleton, J. P. (1997). Assessing the magnitude of the allocentric spatial deficit associated with complete loss of the anterior thalamic nuclei in rats. Behav. Brain Res. 87, 223-232. doi: 10.1016/s01664328(97)02285-7

Wilton, L. A., Baird, A. L., Muir, J. L., Honey, R. C., and Aggleton, J. P. (2001). Loss of the thalamic nuclei for "head direction" impairs performance on spatial memory tasks in rats. Behav. Neurosci. 115, 861-869. doi: 10.1037/0735-7044. 115.4.861

Winter, S. S., Clark, B. J., and Taube, J. S. (2015). Spatial navigation. Disruption of the head direction cell network impairs the parahippocampal grid cell signal. Science 347, 870-874. doi: 10.1126/science.1259591

Wolff, M., Gibb, S. J., and Dalrymple-Alford, J. C. (2006). Beyond spatial memory: the anterior thalamus and memory for the temporal order of a sequence of odor cues. J. Neurosci. 26, 2907-2913. doi: 10.1523/JNEUROSCI. 5481-05.2006

Wolff, M., and Vann, S. D. (2019). The cognitive thalamus as a gateway to mental representations. J. Neurosci. 39, 3-14. doi: 10.1523/JNEUROSCI. 0479-18.2018

Xiao, D., and Barbas, H. (2002). Pathways for emotions and memory II. Afferent input to the anterior thalamic nuclei from prefrontal, temporal, hypothalamic areas and the basal ganglia in the rhesus monkey. Thalamus Related Systems 2, 33-48.

Xiao, D., Zikopoulos, B., and Barbas, H. (2009). Laminar and modular organization of prefrontal projections to multiple thalamic nuclei. Neuroscience 161, 1067-1081. doi: 10.1016/j.neuroscience.2009.04.034

Yu, T., Wang, X., Li, Y., Zhang, G., Worrell, G., Chauvel, P., et al. (2018). Highfrequency stimulation of anterior nucleus of thalamus desynchronizes epileptic network in humans. Brain 141, 2631-2643. doi: 10.1093/ brain/awy187

Conflict of Interest Statement: The authors declare that the research was conducted in the absence of any commercial or financial relationships that could be construed as a potential conflict of interest.

Copyright (C) 2019 Perry and Mitchell. This is an open-access article distributed under the terms of the Creative Commons Attribution License (CC BY). The use, distribution or reproduction in other forums is permitted, provided the original author(s) and the copyright owner(s) are credited and that the original publication in this journal is cited, in accordance with accepted academic practice. No use, distribution or reproduction is permitted which does not comply with these terms. 